\title{
VALIDAÇÃO DA ESCALA DE RAZÃO DAS NECESSIDADES DE FAMILIARES EM UNIDADE DE TERAPIA INTENSIVA ${ }^{1}$
}

Fernanda Helena Morgon ${ }^{2}$ Edinêis de Brito Guirardello ${ }^{3}$

Morgon FH, Guirardello EB. Validação da escala de razão das necessidades de familiares em unidade de terapia intensiva. Rev Latino-am Enfermagem 2004 março-abril; 12(2):198-203.

A hospitalização, por motivo de doença grave e inesperada, pode acarretar um desequilíbrio na estrutura familiar. $A$ família exerce um papel importante na recuperação do paciente e, muitas vezes, suas necessidades são desconhecidas pelo enfermeiro, e pouca ênfase é dada por ele ao atendimento das pessoas. Este estudo teve por objetivo validar, em nível de razão, a escala de necessidades de familiares em Unidade de Terapia Intensiva (UTI). Participaram do estudo 52 familiares que visitaram o seu parente durante a hospitalização na UTI. Utilizaram os métodos psicofísicos de estimação de magnitude e emparelhamento de comprimento de linhas, com cálculo da média geométrica para cada uma das necessidades julgadas, em ambos os métodos. Os resultados mostraram uma concordância significativa, entre os participantes, no julgamento das necessidades, com um coeficiente de correlação de Pearson de $r=0,97$ ( $p<0,0001)$. A relação entre esses métodos pode ser descrita por uma função de potência, confirmando que a escala de necessidades de familiares é válida, estável e consistente.

DESCRITORES: psicofísica; pesos e medidas; família

\section{VALIDATION OF A RATIO SCALE FOR FAMILY NEEDS AT} AN INTENSIVE CARE UNIT

The hospitalization of a close relative due to unexpected critical illness may trigger an unbalance in the patient's family structure. The family plays an important role in the patient's recovery and, often, its needs are unknown by nurses and little emphasis is given to attention to the family's needs. This study aimed to validate a ratio-level scale of family needs at an intensive care unit (ICU). The sample consisted of 52 family members who visited their relatives during ICU hospitalization. Psychophysical methods of magnitude estimation (ME) and cross modality matching (line length) were used, calculating the geometric mean of each need by both methods. The results showed a high agreement on needs among the participants, with a Pearson coefficient of $r=0.97(p<0.0001)$. The relationship between these methods can be described as a power function, confirming that this scale of family needs is valid, stable and consistent.

DESCRIPTORS: psychophysics; weights and measures; family

\section{VALIDACIÓN DE LA ESCALA DE RAZÓN DE LAS NECESIDADES DE FAMILIARES EN UNIDADES DE TERAPIA INTENSIVA}

La hospitalización por motivo de enfermedad grave e inesperada puede acarrear un desequilibrio en la estructura familiar. La familia ejerce un papel importante en la recuperación del paciente pero muchas veces sus necesidades son desconocidas por el enfermero y poca atención es dada por él a la atención de esas necesidades. Este estudio tuvo por objetivo validar la escala de necesidades de familiares en UTI. Participaron del estudio 52 familiares que visitaron su pariente durante la hospitalización en la UTI. Se utilizaron los métodos psicofísicos de estimación de magnitud y de emparejamiento del largo de líneas, calculando la media geométrica para cada una de las necesidades evaluadas con ambos métodos. Los resultados mostraron una concordancia significativa entre los participantes en la evaluación de las necesidades con un coeficiente de correlación de Pearson de $r=0,97$ ( $p<0,0001)$. La relación entre esos métodos puede ser descripta por una función de potencia, confirmando que la escala de necesidades de familiares es válida, estable y consistente.

DESCRIPTORES: psicofisica; pesos y medidas; familia

\footnotetext{
${ }^{1}$ Parte da Dissertação de Mestrado do Programa de Pós-Graduação do Departamento de Enfermagem da Faculdade de Ciências Médicas da Universidade Estadual de Campinas, 2003; ${ }^{2}$ Enfermeira da Unidade de Terapia Intensiva do Hospital de Clínicas da Universidade Estadual de Campinas, Mestre em Enfermagem, e-mail: fehmlili@ig.com.br; ${ }^{3}$ Enfermeira, Professor Doutor da Faculdade de Ciências Médicas da Universidade Estadual de Campinas, e-mail: guirar@fcm.unicamp.br
} 
INTRODUÇÃO

A Unidade de Terapia Intensiva (UTI) caracterizase como um ambiente altamente agressivo e invasivo, em função da intensidade das situações ou da iminência de eventos, tais como: o risco de morte, a necessidade de rotina dinâmica e acelerada da equipe de trabalho, a ausência de controle da iluminação natural e a presença de ruídos sonoros ${ }^{(1)}$.

A hospitalização na UTI, por motivo de doença grave e inesperada, pode acarretar um desequilíbrio na estrutura familiar. A família ocupa um papel importante na recuperação do paciente e, muitas vezes, suas necessidades são desconhecidas pelo enfermeiro ${ }^{(2-3)}$.

As necessidades de familiares são caracterizadas por situações ou eventos de caráter físico e emocional, que podem ser vivenciadas por familiares de pacientes com uma doença grave e inesperada, internados na $U \mathrm{UT}^{(3)}$. Essas necessidades podem ser exemplificadas por situações ou eventos como: saber quem pode dar informação ao familiar, sentir que há esperança de melhora, saber qual tratamento médico está sendo dado e ter orientações gerais sobre a UTI, na primeira visita.

É muito difícil para os familiares, com pouca ou nenhuma experiência, estar adequadamente preparados para uma situação de doença inesperada, quando necessitam tomar decisões com relação ao cuidado de seu familiar, contribuindo para que o impacto cumulativo de uma doença possa resultar no rompimento das atividades diárias dos familiares ${ }^{(4)}$.

O primeiro estudo que se propôs a identificar as necessidades dos familiares de pacientes internados na UTI, desenvolveu um instrumento com 45 necessidades, denominado Critical Care Family Need Inventory (CCFNI) $)^{(3)}$.

Outros estudos utilizaram o CCFNI na íntegra ou parcialmente ${ }^{(5-6)}$. No Brasil, o CCFNI foi adaptado e validado para a cultura brasileira, constituído por uma lista de 43 necessidades, denominado Inventário de Necessidades e Estressores de Familiares de Pacientes Internados em Terapia Intensiva $(\mathrm{INEFTI})^{(7)}$.

Para a maioria dos estudos que utilizou o CCFNI, na íntegra ou parcialmente, um aspecto importante a ser considerado foi o tipo de escala de medida utilizado na aplicação e análise desses instrumentos. A escala de medida utilizada foi a de categoria, que produz dados descritivos ou de mensuração ordinal, nos quais apenas a ordenação entre os valores escalares é admissível. Sendo assim, destaca-se a importância de utilizar outros instrumentos de medida, como os métodos psicofísicos de estimação de magnitude (EM) e emparelhamento de comprimento de linhas (ECL) que produzem medidas em nível de razão. Existem quatro níveis escalares de medida, os quais são: a mensuração nominal, a ordinal, a intervalar e a de razão(8).

A escala de medida nominal é considerada o nível de medida mais simples, pois tem a função apenas de classificar os objetos, ou seja, a única relação entre os objetos é de equivalência, sem qualquer informação quantitativa. Exemplos desse tipo de medida são: a cor de cabelo, o sexo e as características pessoais. A escala de medida em nível ordinal possui a propriedade de ordenar os valores, mas não contém informações sobre a distância entre eles. A intervalar possui a propriedade de ordenar e classificar os objetos, estabelecendo a distância ou diferença entre eles. Já, a escala de medida em nível de razão é considerada o nível mais elevado de mensuração, pois, além de possuir todas as propriedades das escalas anteriores, oferece informações sobre a magnitude de uma situação. Ou seja, é possível verificar a proporção em que um estímulo foi julgado, por ser maior ou menor do que o outro. Para se obter uma escala de medida, em nível de razão, utilizam-se os métodos psicofísicos de EM e ECL.

O método de estimação de magnitude consiste no julgamento pessoal para uma série de situações sociais ou clínicas, de forma que o participante é instruído a designar números que sejam proporcionais à intensidade de sua resposta subjetiva, em relação a uma determinada situação vivenciada por ele ${ }^{(9)}$. O método de emparelhamento de comprimento de linhas consiste no julgamento de uma pessoa para uma série de estímulos, de forma que o participante é solicitado a designar um comprimento de linhas que seja proporcional à intensidade de sua resposta subjetiva ${ }^{(10)}$.

Alguns estudos têm utilizado essa metodologia, abordando diferentes fenômenos como: mensuração da dispnéia $^{(11)}$, mensuração do prestígio do enfermeiro ${ }^{(12)}$, mensuração da dor ${ }^{(13)} \mathrm{e}$ o grau de atenção dirigida do enfermeiro $^{(14)}$. Entretanto, não existem estudos que utilizem tais métodos para mensuração das necessidades de familiares.

\section{OBJETIVOS}

Este estudo tem como objetivo principal validar a escala de necessidades de familiares em UTI, utilizando 
os métodos psicofísicos de estimação de magnitude (EM) e emparelhamento de comprimento de linhas (ECL).

\section{MÉTODO}

\section{Participantes}

Os participantes do estudo foram familiares de pacientes internados na Unidade de Terapia Intensiva de um hospital universitário, no interior do Estado de São Paulo.

Instrumentos

Alguns autores ${ }^{(15-16)}$ recomendam as etapas para o processo de escalonamento: a) identificação de um conjunto de situações; b) definição operacional; c) validação de conteúdo; d) teste piloto e e) aplicação do instrumento.

A primeira etapa desse estudo consistiu na seleção de um conjunto de necessidades, extraídas do estudo de Castro $^{(7)}$, sendo escolhidas aquelas com maiores médias aritméticas. (Figura 1). Para a construção de uma escala em nível de razão, recomenda-se entre 10 a 20 situações ou necessidades que sejam apresentadas ao participante em cada sessão ${ }^{(17)}$, pois esta é uma das etapas que irá constituir o rigor metodológico do processo de escalonamento de estímulos subjetivos ${ }^{(15-17)}$.

1. Sentir que o pessoal do hospital (UTI) se interessa pelo paciente

2. Ver o paciente freqüentemente

3. Ser avisado em casa, sobre mudanças no estado do paciente

4. Saber quem pode dar informação de que eu necessito

5. Saber que tratamento médico está sendo dado ao paciente

6. Ter orientações gerais sobre a UTI, na primeira visita

7. Ter uma boa lanchonete no Hospital

8. Sentir que há esperança de melhora do paciente

9. Ter uma pessoa para orientar, em caso de problemas financeiros

10. Ter horário de visita modificado em casos especiais

11. Saber quais outros profissionais podem me ajudar

12. Conversar sobre a possibilidade de morte do paciente

13. Saber por que determinados tratamentos foram realizados com o paciente

14. Ser comunicado sobre possíveis transferências

15. Estar seguro de que o melhor tratamento possível está sendo dado ao paciente

16. Ter uma pessoa que possa dar informações por telefone

17. Ser acompanhado por profissional, amigo ou familiar, durante a visita na UTI

18. Conversar com a mesma enfermeira todos os dias

19. Ter perguntas respondidas com franqueza

20. Ter alguém que se preocupa com a minha saúde

Figura 1 - Necessidades dos familiares de pacientes internados na UTI, selecionadas em sua forma original do instrumento INEFETI
Em seguida, realizou-se a definição conceitual para cada necessidade selecionada que foi submetida à validação aparente e de conteúdo por seis juízes. O testepiloto foi aplicado a dez familiares de pacientes internados na UTI, para verificar a compreensão com relação às instruções e tarefas a serem executadas por eles, bem como alguns ajustes necessários durante a aplicação do instrumento. É importante destacar que os dados do préteste não foram considerados na amostra.

Procedimentos de coleta de dados

Inicialmente, obteve-se a aprovação do projeto pelo Comitê de Ética em Pesquisa (Parecer no 04/2002). Em seguida, os familiares que atenderam aos critérios de inclusão como: a) internação na UTI por doença grave e inesperada, por infarto agudo do miocárdio, situações de urgência e emergência, traumas ou acidentes, com pelo menos 72 horas de internação na UTI, b) familiares com idade igual ou superior a 18 anos, c) familiares com grau de parentesco como pai, mãe, irmãos, filhos, cônjuge que tenham visitado o paciente no período de hospitalização e tendo participado ou se envolvido com os problemas de saúde de seu parente.

Os familiares foram, então, convidados a participar do estudo e, após anuência e esclarecimento de dúvidas, foram solicitados a assinar o Termo de Consentimento Livre e Esclarecido.

Foram elaboradas instruções específicas para cada modalidade de resposta e apresentadas em folhas separadas, sendo uma para EM (numérica) e outra para ECL (visual). Cada necessidade e sua definição operacional foram apresentadas aos participantes em forma de cartões, um a um, de forma aleatória, para ambos os métodos.

No método de EM, a tarefa dos participantes foi assinalar um número a cada necessidade, que fosse proporcional ao grau de importância atribuído por ele. Para a necessidade ter horário de visita modificado em casos especiais, foi designado, previamente, pelo pesquisador, o valor de referência de 100 pontos. Por exemplo, se o participante julgasse que uma determinada situação tinha sido duas vezes mais importante que o valor de referência (100 pontos) deveria assinalar 200 pontos para aquela necessidade, e assim por diante.

No método de ECL, o participante foi solicitado a designar um comprimento de linhas a cada necessidade 
que fosse proporcional ao valor de referência de $100 \mathrm{~cm}$, designado para a necessidade ter horário de visita modificado em casos especiais. Para essa modalidade de resposta, utilizou-se uma trena. Por exemplo, se o participante julgasse que uma determinada necessidade fosse metade do valor de referência, deveria designar 50 $\mathrm{cm}$ e assim por diante.

Análise estatística

Para a análise dos dados, calculou-se a média geométrica para cada uma das necessidades, em ambos os métodos psicofísicos de EM e ECL. Realizou-se a ordenação de postos para as necessidades e calculou-se o coeficiente de correlação de Pearson entre as estimativas de magnitude e estimativas de emparelhamento de comprimento de linhas.

Para determinar o ajuste da função de potência, envolvendo essas duas modalidades de respostas, sendo uma numérica (EM) e a outra visual (ECL), calculou-se o coeficiente de determinação entre essas modalidades de respostas para cada participante (individualmente). Em seguida, as médias geométricas de cada necessidade, em ambos os métodos, foram projetadas em coordenadas logarítmicas.

\section{RESULTADOS E DISCUSSÃO}

Participaram do estudo 52 familiares, sendo a maioria do sexo feminino $(71,1 \%)$, com média de idade de 41 anos. O grau de parentesco foi: filhos $(34,6 \%)$, seguido de pais (27\%), cônjuges $(21,1 \%)$ e outros $(17,3 \%)$. Todos os familiares eram ingênuos em relação ao método aplicado.

Para escalonar as necessidades dos familiares, calcularam-se as médias geométricas das estimativas de magnitude e emparelhamento de comprimento de linhas para cada uma das necessidades como também a ordenação de suas posições (Tabela 1). Os dados mostraram uma concordância significativa no julgamentos das necessidades, entre os participantes, em ambos os métodos psicofísicos, obtendo um coeficiente de correlação de Pearson ( $r=0,97 ; p<0,0001$ ).
Tabela 1 - Média geométrica das estimativas de magnitude (EM), média geométrica dos emparelhamentos de comprimentos de linhas (ECL) e ordenação de posições (OP) para cada necessidade

\begin{tabular}{|c|c|c|c|c|}
\hline Necessidades de familiares em UTI & EM & OP & ECL & OP \\
\hline $\begin{array}{l}\text { Sentir que o pessoal do hospital (UTI) se } \\
\text { interessa pelo paciente }\end{array}$ & 372,9 & 1 & 401,1 & 1 \\
\hline $\begin{array}{l}\text { Estar seguro de que o melhor tratamento } \\
\text { possível está sendo dado ao paciente }\end{array}$ & 327,8 & 2 & 331,5 & 2 \\
\hline $\begin{array}{l}\text { Sentir que há esperança de melhora do } \\
\text { paciente }\end{array}$ & 316,7 & 3 & 270,9 & 4 \\
\hline $\begin{array}{l}\text { Saber que tratamento médico está sendo } \\
\text { dado ao paciente }\end{array}$ & 269,2 & 4 & 234,6 & 5 \\
\hline $\begin{array}{l}\text { Ter perguntas respondidas com } \\
\text { franqueza }\end{array}$ & 263,7 & 5 & 278,5 & 3 \\
\hline $\begin{array}{l}\text { Saber por que determinados tratamentos } \\
\text { foram realizados ao paciente }\end{array}$ & 214,9 & 6 & 220,5 & 7 \\
\hline $\begin{array}{l}\text { Conversar com a mesma enfermeira } \\
\text { todos os dias }\end{array}$ & 214,8 & 7 & 187,4 & 11 \\
\hline $\begin{array}{l}\text { Ser comunicado sobre possíveis } \\
\text { transferências }\end{array}$ & 214,0 & 8 & 182,9 & 13 \\
\hline $\begin{array}{l}\text { Ter alguém que se preocupa com a } \\
\text { minha saúde }\end{array}$ & 198,9 & 9 & 208,1 & 8 \\
\hline $\begin{array}{l}\text { Ter orientações gerais sobre a UTI na } \\
\text { primeira visita }\end{array}$ & 187,9 & 10 & 230,1 & 6 \\
\hline $\begin{array}{l}\text { Saber quem pode dar informações de } \\
\text { que eu necessito }\end{array}$ & 181,9 & 11 & 162,5 & 16 \\
\hline Ver o paciente freqüentemente & 181,8 & 12 & 194,7 & 10 \\
\hline $\begin{array}{l}\text { Ser avisado em casa sobre mudanças no } \\
\text { estado do paciente }\end{array}$ & 181,2 & 13 & 171,0 & 14 \\
\hline $\begin{array}{l}\text { Saber Quais outros profissionais podem } \\
\text { me ajudar }\end{array}$ & 179,3 & 14 & 198,4 & 9 \\
\hline $\begin{array}{l}\text { Ter uma pessoa que possa dar } \\
\text { informações por telefone }\end{array}$ & 176,0 & 15 & 185,2 & 12 \\
\hline $\begin{array}{l}\text { Ser acompanhado por profissional, } \\
\text { amigo ou familiar, durante a visita na UTI }\end{array}$ & 165,1 & 16 & 169,7 & 15 \\
\hline $\begin{array}{l}\text { Ter uma pessoa para orientar em caso } \\
\text { de problemas financeiros }\end{array}$ & 144,6 & 17 & 135,4 & 18 \\
\hline $\begin{array}{l}\text { Conversar sobre a possibilidade de morte } \\
\text { do paciente }\end{array}$ & 140,7 & 18 & 142,1 & 17 \\
\hline $\begin{array}{l}\text { Ter horário de visita modificado em casos } \\
\text { especiais }\end{array}$ & 100,0 & 19 & 100,0 & 19 \\
\hline Ter uma boa lanchonete no hospital & 50,1 & 20 & 49,3 & 20 \\
\hline
\end{tabular}

As necessidades: sentir que o pessoal do hospital se interessa pelo paciente, estar seguro de que o melhor tratamento possível está sendo dado ao paciente e ter uma boa lanchonete no hospital, ocuparam as mesmas ordenações de postos.

Segundo a Tabela 1, verificam-se diferenças similares nas ordenações de postos entre os métodos de EM e ECL. Por exemplo, para as necessidades: sentir que há esperança de melhora do paciente ocupou a $3^{\text {a }}$ posição (EM) e a 4⿳亠丷 posição (ECL); saber que tratamento médico está sendo dado ao paciente ocupou a 4⿳亠丷a posição (EM) e a $5^{\text {a }}$ posição (ECL); ter perguntas respondidas com franqueza ocupou a $5^{\text {a }}$ posição (EM) e a $3^{\text {a }}$ posição (ECL); saber por que determinados tratamentos foram realizados ao paciente, 6 ? $^{\text {a }}$ posição (EM) e a $7^{\text {a }}$ (ECL); conversar com a mesma enfermeira todos os dias ocupou a $7^{a}$ posição (EM) e a 11르 posição (ECL). De acordo com esses dados, 
as necessidades podem ser agrupadas em necessidades de maior, menor ou grau mediano de importância.

Como citado anteriormente, nos métodos psicofisicos de EM e ECL, que produzem medidas em nível de razão, é possível estabelecer não apenas a diferença entre essas necessidades, mas o quanto, quantitativamente, uma necessidade foi julgada, por ter maior ou menor grau de importância em relação a outra. Por exemplo, a necessidade: sentir que o pessoal do hospital (UTI) se interessa pelo paciente (EM=372,9) foi julgada por ser 1,7 vezes maior do que as necessidades: saber por que determinados tratamentos foram realizados ao paciente $(\mathrm{EM}=214,9)$ e conversar sobre a possibilidade de morte do paciente $(\mathrm{EM}=140,73)$, e duas vezes maior que saber quem pode dar informações de que eu necessito (EM=181,9), e ainda 7,4 vezes maior do que ter uma boa lanchonete no hospital $(E M=50,15)$, e assim sucessivamente.

Para verificar a estabilidade da escala de razão, utilizando-se as duas modalidades de respostas, uma verbal (EM) e outra visual (ECL), as médias geométricas das EM e ECL foram projetadas em coordenadas logarítmicas. A inclinação da linha em coordenadas foi de 0,97 , ajustado esse dado pelo método dos quadrados mínimos, obtendo um coeficiente de determinação de $r^{2}=0,95$ (Figura 2).

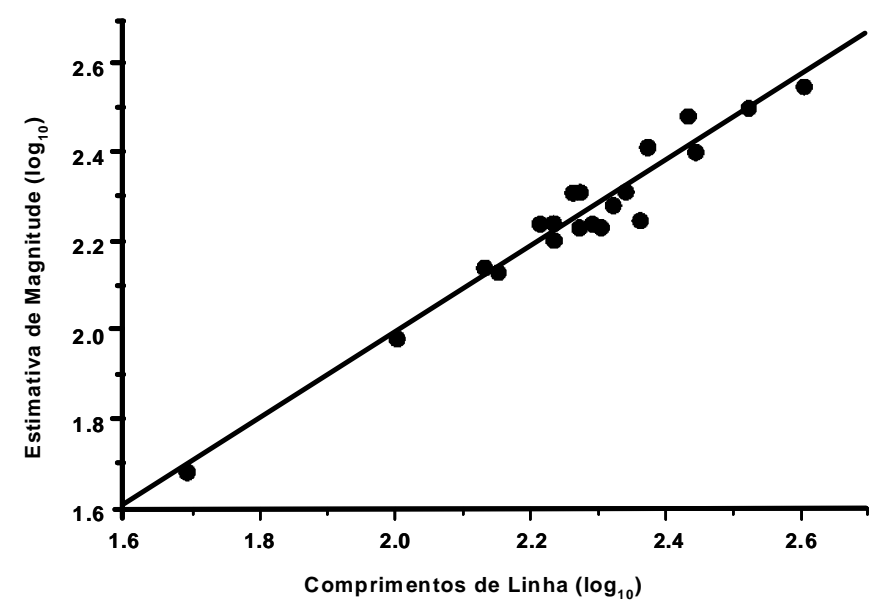

$r=0,97 ;(p<0,0001) ; r^{2}=0,95 ; n^{*}=0,97 ; K=1,20$

Figura 2 - Relação entre os logaritmos das médias geométricas das estimativas de magnitude e dos logaritmos das médias geométricas das estimativas de emparelhamentos de comprimentos de linhas $(n=52)$

Entretanto, como o participante tende a restringir a amplitude de seus ajustamentos, em função da variável que ele controla, essas medidas, em coordenadas invertidas, encontram-se projetadas na Figura 3, ou seja, as médias geométricas do emparelhamento de linhas foram projetadas em coordenadas logarítmicas, em função das correspondentes médias geométricas das estimativas de magnitude para cada necessidade. A inclinação da linha reta em coordenadas invertidas foi 0,98 , ajustado esse dado pelo método dos quadrados mínimos, obtendo um coeficiente de determinação de $r^{2}=0,95$.

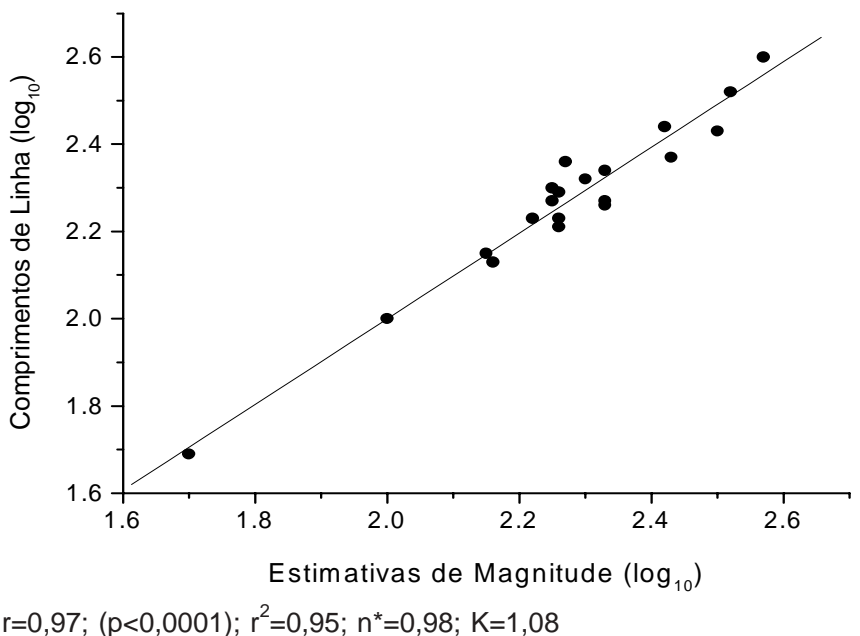

Figura 3 - Relação entre os logaritmos das médias geométricas dos emparelhamentos de comprimentos de linhas e os logaritmos das médias geométricas das estimativas de magnitude $(n=52)$

Esse efeito de regressão foi analisado e comprovada a equivalência entre o expoente empírico e o predito numa tarefa de calibração, envolvendo diretamente duas modalidades de respostas, constituindo-se em forte evidência da validade do método de estimação de magnitudes e, por conseqüência, da função de potência ${ }^{(8)}$.

De acordo com as Figuras 2 e 3 , pode-se observar que os valores médios entre as estimativas de magnitude e as estimativas de comprimentos de linhas, quando projetadas no gráfico, crescem numa mesma proporção, para ambos os métodos, apesar de as retas possuírem uma pequena diferença de inclinação.

\section{CONCLUSÕES}

1. Os familiares dos pacientes julgaram alto grau de importância para a maioria das necessidades apresentadas. Houve uma concordância significativa no julgamento dos participantes, com um coeficiente de correlação de Pearson de 0,97( $p<0,0001)$ para ambos os métodos psicofísicos de EM e ECL. 
2. As necessidades julgadas com maior grau de importância pelos familiares foram: sentir que o pessoal do hospital (UTI) se interessa pelo paciente; estar seguro de que o melhor tratamento possível está sendo dado ao paciente; sentir que há esperança de melhora; saber que tratamento médico está sendo dado e ter perguntas respondidas com franqueza.

3. A ordenação de postos para as diferentes necessidades foram similares em ambos os métodos psicofísicos de EM e ECL. Porém, a sua utilização possibilita estabelecer não somente a ordenação de postos, como também o quanto uma necessidade foi julgada por requerer maior ou menor grau de importância do que outra.

4. As necessidades: sentir que o pessoal do hospital (UTI) se interessa pelo paciente foi julgada como sendo 1,7 vezes maior do que as necessidades saber por que determinados tratamentos foram realizados com o paciente e conversar sobre a possibilidade de morte do paciente, e duas vezes maior do que a necessidade de saber quem pode dar informações de que se necessita, e

\section{REFERÊNCIAS BIBLIOGRÁFICAS}

1. Guirardello EB. Factors in critical care and medical surgical environments that increase the requirements for direct attention. [dissertation]. Madison (WI): School of Nursing, University of Wisconsin, United States; 1993.

2. Leske JS. Needs of relatives of critically ill patients: a followup. Heart \& Lung 1986 March; 15(2):189-93.

3. Molter N. Needs of critically ill patients: a descriptive study. Heart \& Lung 1979 March-April; 3(2):332-9.

4. Neabel B, Fothergill-Bourrbonnais F, Dunning J. Family assessment tools: A review of the literature from 1978-1997. Heart \& Lung 2000 May-June; 29(3):196-209.

5. Daley LK. The perceived immediate needs of families with relatives in the Intensive care setting. Heart \& Lung 1984 May; 13(3):231-7.

6. Mathis M. Personal needs of family members of critically ill patients with and without acute brain injury. J Neurosurg Nurs 1984 February; 16(1):36-44.

7. Castro DS. Estresse e estressores dos familiares de pacientes com traumatismo crânio - encefálico em terapia intensiva. [tese]. Rio de Janeiro (RJ): Escola de Enfermagem Anna Nery/UFRJ; 1999.

8. Stevens SS. To honor Fechener and real his law.Science1961; 133:80-6.

9. Stevens SS. Psychophysics: Introduction to its perceptual, neural and social prospects. New York: John Wiley; 1975.

10. Lodge M. Magnitude scaling: quantitative measurement of opinions. Beverly Hills, CA: Sage; 1981.

11. Nield M, Kim MJ. The reliability of magnitude estimation for dyspnea measurement. Nurs Res 1991 January-February; 40(1):17-9. ainda 7,4 vezes maior do que ter uma boa lanchonete no hospital.

5. A relação entre as estimativas de magnitude e de emparelhamentos de comprimentos de linhas é regida por uma função de potência, e a escala de necessidades de familiares é válida, estável e consistente, com um expoente $\left({ }^{*} n=0,97\right)$ no método de EM e $\left({ }^{*} n=0,98\right)$ no método de ECL, sendo esses expoentes próximos de 1,0, como predito nos experimentos ${ }^{(9)}$.

6. O Enfermeiro está em uma posição singular para identificar essas necessidades, de maneira a implementar uma assistência com qualidade, entendendo-se que a assistência ao paciente na UTI deve estender-se, também, ao familiar do paciente.

7. Os achados desse estudo permitem compreender quais necessidades dos familiares foram julgadas quanto ao seu grau de importância, durante o momento de internação de seu parente na UTI, tendo implicações importantes para a prática do enfermeiro, em relação ao melhor gerenciamento da assistência aos pacientes e familiares.

12. Faleiros Sousa FAE, SILVA JA. Validação da escala de razão de prestígio profissional do enfermeiro através do método de emparelhamento intermodal. Rev Latino-am Enfermagem 1999 Janeiro; 7(1):27-37.

13. Pereira LV, Giuntini PD, Sant'Ana RPM, Faleiros F. Magnitude estimates of pain descriptors. Psychologica 2001; 28:269-76.

14. Guirardello EB, Falleiros Sousa FAE. Mensuração da atenção dirigida do enfermeiro: comparação entre três métodos psicofísicos. Rev Latino-am Enfermagem 2000 Julho; 8(3):108-14.

15. Hinshaw AS. Role attitudes: a measurement problem. In: Hardy ME, Conway ME. Role theory perspectives for health professionals. Michigan: Appleton Century-Crofts; 1978. p. 273-304.

16. Sennott-Miller I, Monaghan C, Hinshaw AS. Magnitude Estimation: Issues and practical application. West J Nurs Res 1988 August; 10(4):414-24.

17. Hamblim RL. Social attitudes: Magnitude measurement and theory. In: Blalock T. Measurement in the social sciences: Theories and strategies. Chicago: Aldine Publishing Company; 1974. p. 61-119. 